\title{
Schwannoma of Foot and Ankle: Seven Case Reports and Literature Review
}

\author{
XINGPEI HAO ${ }^{1}$, DAVID LEVINE ${ }^{1}$, JOON $^{\text {YIM }}{ }^{1}$, CHENFENG $^{2}{ }^{2}$, LEE FIRESTONE ${ }^{1}$, \\ IAN BEISER ${ }^{1}$, ENZO LEONE $^{1}$, KIRK WOELFFER $^{1}$ and GENE MIRKIN ${ }^{1}$ \\ ${ }^{1}$ Foot and Ankle Specialists of the Mid-Atlantic, LLC., Rockville, MD, U.S.A.; \\ ${ }^{2}$ Laboratory of Immunogenetics, National Institute of Allergy and Infectious Diseases, \\ National Institute of Health, Rockville, MD, U.S.A.
}

\begin{abstract}
Background: Schwannoma is most often grown on the trunk, upper and lower extremities, and head and neck, but rarely on the foot. This study aimed to reveal clinical presentations, histopathology and treatment options for schwannoma of the foot. Materials and Methods: Seven schwannomas out of 174 soft-tissue tumors on the foot and ankle were retrieved from our Institute in a 3-year period, and 42 schwannomas on the foot and ankle in the literature in a 30-year period were reviewed. Results: The incidence of schwannoma of foot was found to be $4.0 \%$. The patient age ranged from 8 to 84 years, with a mean of 47.4 years. More than $80 \%$ of tumors were located on the ankle, heel and plantar aspect. Overall, $77.6 \%$ of patients complained about a painful mass. Magnetic resonance imaging revealed a well-circumscribed, round or ovoid mass with iso-intensity signal compared with surrounding neuromuscular tissues on T1-weighted images and hyper-intensity signal on T2. Forty-eight out of 49 patients were treated with surgical excision or enucleation without recurrence in follow-up from 2 months to 4 years. Histologically, schwannoma was composed of hypercellular Antoni A zone with palisaded spindle cells with strong immunostaining for S-100 and hypocellular Antoni $B$ zone with vascularization in myxoid stroma. Conclusion: Schwannoma of the foot and ankle is a rare, painful, indurated tumor. Magnetic resonance imaging reveals the location, size, texture and relationships with surrounding neuromuscular structures. Surgical excision is the primary treatment option with excellent outcome.
\end{abstract}

Correspondence to: Dr. Xingpei Hao. 1600 Gude Drive, Suite 205, Rockville, MD 20852, U.S.A. Tel: +1 3014174372, Fax: +1 3019337137, e-mail: xhao@footandankle-usa.com; Dr. Gene Mirkin 10901 Connecticut Avenue, Suite 200, Kensington, MD 20895 U.S.A. Tel: +1 3019492000, Fax: +1 3019492002, e-mail: gmirkin@footandankle-usa.com

Key Words: Ankle, excision, foot, MRI, pathology, schwannoma.
Schwannoma, also called neurilemoma, derived from Schwann cells in the peripheral nerves, is a solid, slowgrowing tumor. It was first reported by Verocay in 1910 (1), and was named 'schwannoma' by Masson in 1932 (2). Schwannoma accounts for about 5\% benign tumors as revealed by an analysis of 18,677 benign soft-tissue tumors by Kransdorf (3) and others (4). It frequently occurs in the trunk, flexor side of upper and lower extremities, head and neck $(3,5,6)$, especially in the eighth cranial vestibular nerves (7-9), but is uncommon on the foot and ankle (10-12). Toepfer et al. observed 11 (10.5\%) neurinomas, including both schwannoma and neurofibroma, out of 104 benign softtissue tumors of the foot and ankle at a university tumor institute between 1997 and 2015 (13). Kehoe et al. recorded 12 out of $104(11.5 \%)$ peripheral nerve tumors on the foot in a 32-year period (7), and Carvajal et al. reported eight out of $87(9.2 \%)$ schwannomas were located on the foot in a 16year period observation (14). In 1969, Das Gupta et al. analyzed 303 schwannomas with none of them being on the foot (5), whereas in 1998 with Chaudhuri, they reported four out of 104 schwannomas to be found on the foot (15). Odom et al. reviewed 557 schwannomas and noted 19 (2.93\%) involving the foot (16). Most published results were reports of single case or small case series. Here, we report seven cases of schwannoma on the foot and ankle from our Institute in a 3 -year period, and further review 42 cases in the literature from 1988 to date in a 30-year period, with the aim of describing its clinical manifestations, diagnosis, differential diagnosis and treatment options.

\section{Patients and Methods}

All tumors on the foot and ankle were retrospectively analyzed from Foot and Ankle Specialists of Mid-Atlantic (FASMA) in a 3-year period from Sept 1st 2015 to Aug 30th, 2018. FASMA comprises 63 podiatrists located in Washington DC, Maryland, Virginia, North Carolina and Pennsylvania who treat various foot and ankle conditions. All pathology slides of schwannomas were reviewed with confirmation of the original diagnoses (XPH). Clinicopathological 
Table I. Clinicopathological characteristics of study patients with schwannoma on the foot and ankle.

\begin{tabular}{|c|c|c|c|c|c|c|c|}
\hline Case & $\begin{array}{l}\text { Age, } \\
\text { years }\end{array}$ & Gender & Site & Clinical presentation & $\begin{array}{l}\text { Histological } \\
\text { features }\end{array}$ & Treatment & Recurrence \\
\hline 1 & 52 & Male & $\begin{array}{l}\text { Right } \\
\text { medial } \\
\text { ankle }\end{array}$ & $\begin{array}{l}\text { A mass associated with paresthesias for } 1 \text { year. } \\
\text { Ultrasound: An anechoic, oval shaped mass, } 4 \mathrm{~cm} \text {. } \\
\text { MRI: A mass with iso-intensity signal relative to } \\
\text { surrounding muscles on T1 and hyperintensity } \\
\text { signal on T2-weighted images (Figure 1). }\end{array}$ & $\begin{array}{l}\text { Typical } \\
\text { schwannoma } \\
\text { S- } 100^{+}\end{array}$ & $\begin{array}{l}\text { Surgical } \\
\text { enucleation } \\
\text { from } \\
\text { tibial nerve }\end{array}$ & $\begin{array}{c}\text { None } \\
\text { (14 months) }\end{array}$ \\
\hline 2 & 66 & Male & $\begin{array}{l}\text { Right } \\
\text { medial } \\
\text { plantar } \\
\text { heel }\end{array}$ & $\begin{array}{l}\text { A painful nodular mass for } 2 \text { months. } \\
\text { Tender with direct palpation. } \\
\text { MRI: A } 0.38 \mathrm{~cm} \text { nodule of increased signal } \\
\text { intensity on T2-weighted images } \\
\text { in the subdermal region }\end{array}$ & $\begin{array}{l}\text { Typical } \\
\text { schwannoma } \\
\text { S- } 100^{+} ; \alpha \mathrm{SMA}^{-} \\
\text {(Figure 2) }\end{array}$ & $\begin{array}{l}\text { Surgical } \\
\text { excision }\end{array}$ & $\begin{array}{c}\text { None } \\
\text { (12 months) }\end{array}$ \\
\hline 3 & 25 & Female & $\begin{array}{l}\text { Left } 3 \text { rd } \\
\text { interdigital } \\
\quad \text { space }\end{array}$ & $\begin{array}{l}\text { Discolored, painful neoplasm for } \\
3 \text { months, } 0.5 \mathrm{~cm}\end{array}$ & $\begin{array}{l}\text { Compound melanocytic } \\
\text { nevus (CMN) with } \\
\text { underlying schwannoma } \\
\text { S-100+ and HMB- } 45+ \\
\text { for both neval cells of } \\
\text { CMN and the spindle } \\
\text { cells of schwannoma. } \\
\text { aSMA- for both } \\
\text { components }\end{array}$ & $\begin{array}{l}\text { Opted for } \\
\text { observation } \\
\text { after biopsy }\end{array}$ & $\begin{array}{l}\text { None } \\
\text { (12 months) } \\
\text { Lost to } \\
\text { follow-up } \\
\text { thereafter }\end{array}$ \\
\hline 4 & 75 & Female & $\begin{array}{l}\text { Right } \\
\text { plantar }\end{array}$ & $\begin{array}{l}\text { A soft tissue mass for decades, much } \\
\text { tender with weight bearing for several years. } \\
\text { Ultrasound: A well-defined } \\
\text { hypoechoic mass, } 2.5 \mathrm{~cm}\end{array}$ & $\begin{array}{l}\text { Typical schwanoma } \\
\mathrm{S}^{-} 100^{+}, \alpha \mathrm{SMA}^{-}\end{array}$ & $\begin{array}{l}\text { Surgical } \\
\text { excision }\end{array}$ & $\begin{array}{c}\text { None } \\
\text { (38 months) }\end{array}$ \\
\hline 5 & 71 & Male & $\begin{array}{l}\text { Right } 2 \mathrm{nd} \\
\text { interdigital } \\
\text { space }\end{array}$ & $\begin{array}{l}\text { A painful mass for months. } \\
\text { MRI: A moderately enhancing } \\
\text { circumscribed bilobed soft tissue mass, } \\
3.2 \times 2.2 \times 1.5 \mathrm{~cm}\end{array}$ & $\begin{array}{c}\text { Ancient type } \\
\text { schwannoma with } \\
\text { atypical nuclei } \\
\text { S- } 100^{+} ; \alpha \mathrm{SMA}^{-}, \mathrm{CD} 34-\end{array}$ & $\begin{array}{l}\text { Surgical } \\
\text { excision }\end{array}$ & $\begin{array}{c}\text { None } \\
\text { (36 months) }\end{array}$ \\
\hline 6 & 36 & Male & $\begin{array}{l}\text { Left dorsal } \\
\text { to the 4th and } \\
5 \text { th metatarsals }\end{array}$ & $\begin{array}{c}\text { A painful mass for } \\
2 \text { months, } 1.2 \times 1.5 \mathrm{~cm}\end{array}$ & $\begin{array}{c}\text { Typical schwannoma } \\
\text { S- } 100^{+} ; \alpha \mathrm{SMA}^{-}, \mathrm{CD} 34^{-}\end{array}$ & $\begin{array}{l}\text { Surgical } \\
\text { excision }\end{array}$ & $\begin{array}{c}\text { None } \\
\text { (28 months) }\end{array}$ \\
\hline 7 & 34 & Female & $\begin{array}{l}\text { Left } \\
\text { posterior } \\
\text { ankle }\end{array}$ & $\begin{array}{l}\text { A firm, tender, ganglion cyst-like } \\
\text { mass for } 2 \text { years, } 1 \mathrm{~cm}\end{array}$ & $\begin{array}{l}\text { Typical schwannoma } \\
\qquad \mathrm{S} 100^{+} ; \alpha \mathrm{SMA}^{-}\end{array}$ & $\begin{array}{l}\text { Surgical } \\
\text { excision }\end{array}$ & $\begin{array}{c}\text { None } \\
\text { (12 months) }\end{array}$ \\
\hline
\end{tabular}

IHC: Immunohistochemistry; $\alpha$ SMA: alpha smooth muscle actin; CD: cluster of differentiation; MRI: magnetic resonance imaging. Typical schwannoma is histologically composed of hypercellular Antoni A with elongated spindle or ovoid Schwann cells forming bundles and hypocellular Antoni B with fewer spindle or ovoid cells admixed with vascular vessels within the loose matrix with myxoid changes.

features were retrieved from FASMA's database, analyzed by one observer (XPH) and were further confirmed by the referring podiatrists.

\section{Results}

Clinicopathological characteristics of schwannoma of foot and ankle. In total, seven schwannomas out of 174 benign soft-tissue tumors were diagnosed in the foot and ankle areas in a 3-year period. The incidence of schwannoma of the foot and ankle was $4.0 \%$. The clinicopathological characteristics of seven schwannomas are shown in Table I.

English literature of 42 schwannomas involving the foot and ankle from 1988 for a 30-year period were reviewed (Table II). Only cases with clear age, gender, size, site, MRI, histology report, treatment, follow-up etc. were included for further analysis. Other reports without detailed clinical information were not included. This brought the number to a total of 49 patients including the current seven cases, making this the most detailed analysis of schwannomas involving the foot and ankle. The clinicopathological features were analyzed based on these 49 cases.

Clinically, most of the patients $(38 / 49,77.6 \%)$ complained of painful mass on the foot or ankle with or without weakness or paresthesia. Three patients exhibited tarsal tunnel syndrome due to involvement of the tibial nerve in the tarsal tunnel. The duration from the symptom onset to surgery ranged from 2 months to 48 years, with a mean and median of 7.3 and 3 years, respectively. Both males and females were equally affected (males to females: 1:1). The 
Table II. Review of clinicopathological features of patients with schwannoma on the foot and ankle reported in literature.

\begin{tabular}{|c|c|c|c|c|c|c|c|}
\hline $\begin{array}{l}\text { Author (Ref), } \\
\text { year }\end{array}$ & $\begin{array}{l}\text { Age, } \\
\text { years }\end{array}$ & Gender & Site & Clinical presentation & $\begin{array}{l}\text { Histological } \\
\text { feature }\end{array}$ & Treatment & Recurrence \\
\hline $\begin{array}{l}\text { Zuckerman } \\
\text { et al. }(23), 1988\end{array}$ & 73 & M & $\begin{array}{l}\text { Left medial } \\
\text { and plantar }\end{array}$ & $\begin{array}{l}\text { An asymptomatic mass for } 40 \text { years, } \\
\text { increased pain for } 2 \text { months, } 2.5 \times 2.5 \mathrm{~cm}\end{array}$ & Typical & $\begin{array}{l}\text { Surgical excision from } \\
\text { medial plantar nerve }\end{array}$ & $\begin{array}{l}\text { None } \\
(30 \text { months })\end{array}$ \\
\hline $\begin{array}{l}\text { Buenger } \\
\text { et al. }(20), 1993\end{array}$ & 53 & M & $\begin{array}{l}\text { Left dorsal } \\
\text { foot and ankle }\end{array}$ & $\begin{array}{l}\text { Several painful, slowly growing nodules for } \\
30 \text { years after sustaining several injuries } \\
\text { to the involved extremity, } 0.5-2.5 \mathrm{~cm}\end{array}$ & Typical & Surgical excision & N/A \\
\hline $\begin{array}{l}\text { Ikushima } \\
\text { et al. (24), } 1999\end{array}$ & 8 & M & $\begin{array}{l}\text { Left medial } \\
\text { and plantar }\end{array}$ & $\begin{array}{l}\text { Asymptomatic, soft, immobile, } \\
\text { non-tender mass, } 6 \times 4 \mathrm{~cm}\end{array}$ & $\begin{array}{l}\text { Plexiform } \\
\text { schwannoma } \\
\text { with almost } \\
\text { uniform } \\
\text { cellular } \\
\text { Antoni A }\end{array}$ & Surgical excision & $\begin{array}{c}\text { None } \\
\text { (24 months) }\end{array}$ \\
\hline $\begin{array}{l}\text { Ritte and Elston } \\
(25), 2001\end{array}$ & 19 & M & Left dorsum & $\begin{array}{l}\text { A solitary, pediculated, fleshy } \\
\text { nodule for } 1 \text { year, } 1 \mathrm{~cm}\end{array}$ & Typical & Surgical excision & $\begin{array}{c}\text { None } \\
(2 \text { months })\end{array}$ \\
\hline $\begin{array}{l}\text { Torossian } \\
\text { et al. }(18), 2001\end{array}$ & 30 & M & Right heel & $\begin{array}{l}\text { Incompletely excised small schwannoma } \\
10 \text { years earlier. } \\
\text { Slow-growing mass with difficult walking } \\
\text { for two year, } 7.5 \times 5.5 \times 5 \mathrm{~cm} \text {, ulcerated, } \\
\text { irregular, ovoid, solid, well circumscribed }\end{array}$ & Typical & $\begin{array}{l}\text { Surgical excision } \\
\text { and reconstruction } \\
\text { with a medial } \\
\text { plantar flap }\end{array}$ & $\begin{array}{l}\text { None } \\
(8 \text { months })\end{array}$ \\
\hline $\begin{array}{l}\text { Odom } \\
\text { et al. }(16), 2001\end{array}$ & 74 & $\mathrm{~F}$ & Left heel & $\begin{array}{l}\text { A painful mass for } 5 \text { years. } \\
\text { MRI: A } 1.5 \mathrm{~cm} \text { in diameter, round, } \\
\text { circumscribed mass involving } \\
\text { the medial plantar nerve }\end{array}$ & Typical & Surgical excision & $\begin{array}{c}\text { None } \\
(18 \text { months })\end{array}$ \\
\hline Still (26), 2001 & 55 & $\mathrm{~F}$ & $\begin{array}{l}\text { Left foot } \\
\text { bottom }\end{array}$ & $\begin{array}{l}\text { A slowly enlarging painful mass for } 2 \text { years. } \\
\text { MRI: An oval shaped mass associated } \\
\text { with medial plantar nerve, } 3.8 \times 2.0 \mathrm{~cm}\end{array}$ & Typical & $\begin{array}{c}\text { Surgical } \\
\text { enucleation }\end{array}$ & $\begin{array}{l}\text { None } \\
(19 \text { months })\end{array}$ \\
\hline \multirow[t]{2}{*}{$\begin{array}{l}\text { Marui } \\
\text { et al. }(27), 2004\end{array}$} & 84 & $\mathrm{~F}$ & Right heel & $\begin{array}{l}\text { A painful mass for } 10 \text { years. } \\
\text { MRI: A soft-tissue mass within the } \\
\text { medial plantar nerve. } 1.8 \times 1 \times 1 \mathrm{~cm}\end{array}$ & Typical & Surgical excision & $\begin{array}{c}\text { None } \\
(15 \text { months })\end{array}$ \\
\hline & 53 & M & Right heel & $\begin{array}{l}\text { Painful nodule for } 8 \text { years. } \\
\text { MRI: A soft tissue tumor associated } \\
\text { with the first branch of the lateral } \\
\text { plantar nerve, } 1 \times 0.9 \times 1 \mathrm{~cm}\end{array}$ & $\begin{array}{l}\text { Typical } \\
\text { S- } 100^{+}\end{array}$ & $\begin{array}{c}\text { Surgical } \\
\text { enucleation }\end{array}$ & $\begin{array}{c}\text { None } \\
(16 \text { months })\end{array}$ \\
\hline $\begin{array}{l}\text { Mangrulkar } \\
\text { et al. (17), } 2007\end{array}$ & 37 & M & Left foot & $\begin{array}{l}\text { A painful, multinodular, firm mass for years. } \\
\text { MRI: A } 14 \times 5 \times 8 \mathrm{~cm} \text { soft tissue mass } \\
\text { involving posterior tibial nerve, medial } \\
\text { and lateral plantar nerves }\end{array}$ & Typical & $\begin{array}{l}\text { Surgical excision. } \\
\text { Surgical excision } \\
\text { again due to } \\
\text { recurrence at } \\
\quad 9 \text { months }\end{array}$ & $\begin{array}{l}\text { Recurrence } \\
\text { at } 9 \text { months, } \\
\text { none after } \\
\text { resection at } \\
24 \text {-month } \\
\text { follow-up }\end{array}$ \\
\hline $\begin{array}{l}\text { Ioannou } \\
\text { et al. }(28), 2009\end{array}$ & 29 & M & Right ankle & $\begin{array}{c}\text { A slowly growing mass with mild } \\
\text { pain and paresthesia for } 6 \text { months. } \\
\text { MRI: A multinodular, inhomogeneous } \\
\text { lesion, } 6 \times 4 \times 2.8 \mathrm{~cm}\end{array}$ & $\begin{array}{c}\text { Plexiform } \\
\text { schwannoma } \\
\text { S-100+, actin } \\
\text { desmin } \\
\text { de }\end{array}$ & $\begin{array}{l}\text { Surgical excision } \\
\text { from posterior } \\
\text { tibial nerve }\end{array}$ & $\begin{array}{c}\text { None } \\
\text { (28 months) }\end{array}$ \\
\hline $\begin{array}{l}\text { Kwok } \\
\text { et al. (29), } 2009\end{array}$ & 42 & M & $\begin{array}{l}\text { Left medial } \\
\text { heel }\end{array}$ & $\begin{array}{l}\text { Left medial heel pain radiating to } \\
\text { the lateral sole for } 6 \text { months, Tinel }{ }^{+} \text {. } \\
\text { Ultrasonography and MRI: A neurogenic } \\
\text { tumor inside the tarsal tunnel, } 1-\mathrm{cm}\end{array}$ & Typical & $\begin{array}{l}\text { Surgical excision } \\
\text { from the first } \\
\text { branch of the } \\
\text { lateral plantar nerve. }\end{array}$ & $\begin{array}{c}\text { None } \\
(14 \text { months })\end{array}$ \\
\hline $\begin{array}{l}\text { Mendeszoon } \\
\text { et al. }(30), 2009\end{array}$ & 56 & $\mathrm{~F}$ & Left foot & $\begin{array}{l}\text { Left foot pain and swelling for } 3 \text { years. } \\
\text { MRI: A large cystic lesion, } 8 \times 2.8 \times 3.1 \mathrm{~cm}\end{array}$ & Typical & Surgical excision & $\begin{array}{c}\text { None } \\
(5 \text { months })\end{array}$ \\
\hline $\begin{array}{l}\text { Jacobson } \\
\text { et al. }(21), 2011\end{array}$ & 65 & M & $\begin{array}{l}\text { Left } \\
\text { plantarlateral } \\
\text { aspect } \\
\text { of forefoot }\end{array}$ & $\begin{array}{l}\text { A large, soft, nontender, partially } \\
\text { pedunculated soft tissue mass for } \\
48 \text { years following minor trauma. } \\
\qquad 10.1 \times 6.8 \times 3.5 \mathrm{~cm}\end{array}$ & $\begin{array}{l}\text { Plexiform } \\
\text { schwannoma } \\
\text { S- } 100^{+}\end{array}$ & Surgical excision & $\begin{array}{c}\text { None } \\
(28 \text { months })\end{array}$ \\
\hline Carvajal & 33 & M & N/A & Left painful mass, $2 \mathrm{~cm}$, paresthesias & Typical & Surgical excision & N/A \\
\hline et al. (14), 2011 & 45 & $\mathrm{~F}$ & N/A & $\begin{array}{c}\text { Left painful mass, } 2 \mathrm{~cm} \\
\text { Tinel+ }\end{array}$ & $\begin{array}{l}\text { Schwannoma } \\
\text { associated with } \\
\text { neurofibro- } \\
\text { matosis-1 }\end{array}$ & Surgical excision & Recurrence \\
\hline
\end{tabular}


Table II. Continued

\begin{tabular}{|c|c|c|c|c|c|c|c|}
\hline $\begin{array}{l}\text { Author (Ref), } \\
\text { year }\end{array}$ & $\begin{array}{l}\text { Age, } \\
\text { years }\end{array}$ & Gender & Site & Clinical presentation & $\begin{array}{l}\text { Histological } \\
\text { feature }\end{array}$ & Treatment & Recurrence \\
\hline & 37 & $\mathrm{~F}$ & N/A & $\begin{array}{c}\text { Left painful mass, } 2.3 \mathrm{~cm} \\
\text { Tinel+ }\end{array}$ & Typical & Surgical excision & N/A \\
\hline & 67 & $\mathrm{~F}$ & N/A & Right painful mass, $2.5 \mathrm{~cm}$, Tinel+ & Typical & Surgical excision & N/A \\
\hline $\begin{array}{l}\mathrm{Li} \\
\text { et al. (31), } 2011\end{array}$ & 19 & $\mathrm{~F}$ & $\begin{array}{l}\text { Left plantar } \\
\text { aspect }\end{array}$ & $\begin{array}{l}\text { Progressive pain for } 18 \text { months. } \\
\text { MRI: Three nodules, } 0.5 \times 0.5 \times 0.8 \mathrm{~cm} \text {, } \\
0.7 \times 1.0 \times 1.2 \mathrm{~cm}, 1.0 \times 1.1 \times 1.5 \mathrm{~cm}\end{array}$ & $\begin{array}{c}\text { Plexiform } \\
\text { schwannoma }\end{array}$ & $\begin{array}{l}\text { Surgical excision } \\
\text { from plantar } \\
\text { digital nerve }\end{array}$ & $\begin{array}{l}\text { None } \\
\text { (30 months) }\end{array}$ \\
\hline $\begin{array}{l}\text { Milnes and } \\
\text { Pavier (32), } 2012\end{array}$ & 73 & $\mathrm{~F}$ & Left ankle & $\begin{array}{l}\text { An exquisitely painful, solid and } \\
\text { immobile mass for } 8 \text { years. Tinel+. } \\
\text { Tarsal tunnel syndrome. } \\
\text { MRI: A solid mass, } 3 \mathrm{~cm}\end{array}$ & Typical & $\begin{array}{l}\text { Surgical } \\
\text { enucleation from } \\
\text { tibial nerve }\end{array}$ & $\begin{array}{l}\text { None } \\
\text { (6 months) }\end{array}$ \\
\hline \multirow[t]{2}{*}{$\begin{array}{l}\text { Schweitzer } \\
\text { et al. (33), } 2013\end{array}$} & 65 & $\mathrm{~F}$ & $\begin{array}{l}\text { Left } \\
\text { posteromedial } \\
\text { ankle }\end{array}$ & $\begin{array}{l}\text { Painful masses for } 5 \text { years. } \\
\text { MRI: Five, well-defined, } \\
\text { fusiform-shaped masses } 0.5 \text { to } 2 \mathrm{~cm}\end{array}$ & Typical & Surgical excision & $\begin{array}{c}\text { None } \\
(12 \text { months })\end{array}$ \\
\hline & 63 & M & Right ankle & $\begin{array}{l}\text { An asymptomatic "lump" for } \\
30 \text { years, increased pain for } 3 \text { months. } \\
\text { MRI: } 3 \text { separate masses, } 0.8 \text { to } 2.8 \mathrm{~cm}\end{array}$ & $\begin{array}{l}\text { Typical } \\
\text { S- } 100^{+}\end{array}$ & $\begin{array}{l}\text { Surgical } \\
\text { enucleation }\end{array}$ & $\begin{array}{c}\text { None } \\
\text { (12 months) }\end{array}$ \\
\hline \multirow[t]{3}{*}{$\begin{array}{l}\text { Kellner } \\
\text { et al. (34), } 2014\end{array}$} & N/A & N/A & Left ankle & $\begin{array}{l}\text { A painful mass for } 3 \text { years, } 2 \mathrm{~cm} \text {, Tinel+. } \\
\text { MRI: A well-circumscribed, enhancing } \\
\text { lesion in the region of the tarsal tunnel }\end{array}$ & Typical & $\begin{array}{l}\text { Surgical resection } \\
\text { from tibial nerve }\end{array}$ & N/A \\
\hline & N/A & N/A & $\begin{array}{l}\text { Left medial } \\
\text { ankle }\end{array}$ & $\begin{array}{l}\text { A mass for } 4 \text { years, } 2 \mathrm{~cm} \text {. } \\
\text { MRI: A well-circumscribed, noninvasive } \\
\text { mass located on the calcaneal branch } \\
\text { of the posterior tibial nerve }\end{array}$ & Typical & Surgical excision & N/A \\
\hline & $\mathrm{N} / \mathrm{A}$ & N/A & Left heel & A mobile mass for 20 years, $1.5 \mathrm{~cm}$ & Typical & Surgical excision & N/A \\
\hline $\begin{array}{l}\text { Kallini and } \\
\text { Khachemoune } \\
(35), 2014\end{array}$ & 25 & M & $\begin{array}{l}\text { Left lateral } \\
\text { heel }\end{array}$ & $\begin{array}{l}\text { A painless, flesh-colored, soft, indurated } \\
\text { nodule with mild hyperpigmentation } \\
\text { for at least } 1 \text { year, } 3 \times 3 \mathrm{~cm}\end{array}$ & Typical & Surgical excision & N/A \\
\hline $\begin{array}{l}\text { Hallahan } \\
\text { et al. (36), } 2014\end{array}$ & 54 & $\mathrm{~F}$ & Left ankle & $\begin{array}{l}\text { A firm and palpable mass for } \\
10 \text { years, tarsal tunnel syndrome. } \\
\text { MRI: A mass, } 3.4 \times 3.0 \times 3.0 \mathrm{~cm}\end{array}$ & $\begin{array}{l}\text { Typical } \\
\text { S- } 100^{+}\end{array}$ & Surgical excision & $\begin{array}{l}\text { None } \\
\text { (9 months) }\end{array}$ \\
\hline \multirow[t]{2}{*}{$\begin{array}{l}\text { Mohammed } \\
\text { et al. (37), } 2014\end{array}$} & 38 & $\mathrm{~F}$ & Right heel & $\begin{array}{l}\text { A painful, firm, immobile mass for } \\
30 \text { years, increased size } 1 \text { year ago. } \\
\text { MRI: A serpiginous, lobulated mass, } \\
2.1 \times 1.5 \times 2.3 \mathrm{~cm}\end{array}$ & $\begin{array}{l}\text { Plexiform } \\
\text { schwannoma } \\
\text { S- } 100^{+}\end{array}$ & Surgical excision & $\begin{array}{l}\text { None } \\
(10 \text { months })\end{array}$ \\
\hline & 11 & M & Right plantar & $\begin{array}{l}\text { A painful mass for } 1 \text { year. } \\
\text { MRI: A multilobulated mass } \\
\text { in the plantar soft tissues } \\
\text { of the forefoot, } 3.9 \times 3.2 \times 2.4 \mathrm{~cm}\end{array}$ & $\begin{array}{l}\text { Plexiform } \\
\text { schwannoma } \\
\text { S-100+ }\end{array}$ & Surgical excision & $\begin{array}{c}\text { None } \\
\text { (36 months) }\end{array}$ \\
\hline $\begin{array}{l}\text { Judd } \text { et al. } \\
\text { (22), } 2014\end{array}$ & 51 & M & $\begin{array}{l}\text { Right medial } \\
\text { ankle }\end{array}$ & $\begin{array}{l}\text { A soft-tissue mass for } \\
2 \text { years after injury of ankle. } \\
\text { MRI: A lobular cystic lesion, } \\
2.5 \times 2.8 \times 1.5 \mathrm{~cm}\end{array}$ & $\begin{array}{l}\text { Typical } \\
\text { S- } 100^{+}\end{array}$ & $\begin{array}{l}\text { Surgical excision } \\
\text { from small } \\
\text { branches of the } \\
\text { posterior tibial nerve }\end{array}$ & N/A \\
\hline $\begin{array}{l}\text { Nishio } \\
\text { et al. (38), } 2015\end{array}$ & 32 & $\mathrm{~F}$ & Right ankle & $\begin{array}{l}\text { A slowly growing, painful mass for } 5 \text { years. } \\
\text { MRI: Multiple nodular lesions with } \\
\text { heterogeneous high signal intensity on } \\
\text { T2-weighted sequences, } 0.2 \text { to } 3 \mathrm{~cm}\end{array}$ & $\begin{array}{c}\text { Plexiform } \\
\text { schwannoma }\end{array}$ & $\begin{array}{l}\text { Surgical } \\
\text { intracapsular } \\
\text { enucleation }\end{array}$ & N/A \\
\hline $\begin{array}{l}\text { Min } \\
\text { et al. (39), } 2015\end{array}$ & 38 & M & $\begin{array}{l}\text { Left middle } \\
\text { plantar }\end{array}$ & $\begin{array}{l}\text { Persistent, pin-prick painful } \\
\text { soft-tissue mass for } 5 \text { years, Tinel+. } \\
\text { MRI: Two fusiform masses along the medial } \\
\text { plantar nerve, } 3.2 \times 2.3 \times 2.2 \mathrm{~cm}, 1 \times 0.9 \times 0.8 \mathrm{~cm}\end{array}$ & Typical & Surgical excision & $\begin{array}{c}\text { None } \\
(2 \text { months })\end{array}$ \\
\hline \multirow[t]{2}{*}{$\begin{array}{l}\text { Albert } \\
\text { et al. (40), } 2017\end{array}$} & 26 & $\mathrm{~F}$ & Heel & $\begin{array}{l}\text { Lower extremity calf tenderness, pain, and } \\
\text { progressive weakness. Ultrasonography: } \\
\text { A hypoechoic soft tissue mass, } 3 \mathrm{~cm} \\
\text { MRI: A soft-tissue mass along the } \\
\text { course of the sural nerve }\end{array}$ & Typical & Surgical excision & N/A \\
\hline & 22 & M & Heel & $\begin{array}{l}\text { Tenderness and palpable mass on the } \\
\text { lateral aspect of the heel inferior. }\end{array}$ & Typical & Surgical excision & N/A \\
\hline
\end{tabular}


Table II. Continued

\begin{tabular}{|c|c|c|c|c|c|c|c|}
\hline $\begin{array}{l}\text { Author (Ref), } \\
\text { year }\end{array}$ & $\begin{array}{l}\text { Age, } \\
\text { years }\end{array}$ & Gender & Site & Clinical presentation & $\begin{array}{l}\text { Histological } \\
\text { feature }\end{array}$ & Treatment & Recurrence \\
\hline \multicolumn{8}{|c|}{$\begin{array}{l}\text { Ultrasonography: A hypoechoic solid soft } \\
\text { tissue nodular mass, } 1.1 \mathrm{~cm} \times 0.6 \mathrm{~cm}\end{array}$} \\
\hline \multicolumn{8}{|c|}{$\begin{array}{l}\text { MRI: A peripherally enhancing soft-tissue mass } \\
\text { along the course of the sural nerve }\end{array}$} \\
\hline & 66 & $\mathrm{~F}$ & Ankle & \multicolumn{2}{|c|}{$\begin{array}{l}\text { A painful mass at the level of tarsal tunnel, Tinel+. Typical } \\
\text { MRI: A solid, polylobulated mass within } \\
\text { the subcutaneous fat, } 1.9 \mathrm{~cm}\end{array}$} & Surgical excision & N/A \\
\hline $\begin{array}{l}\text { Muratori } \\
\text { et al. }(41), 2017\end{array}$ & 65 & $\mathrm{~F}$ & $\begin{array}{l}\text { Right medial } \\
\text { and plantar }\end{array}$ & $\begin{array}{l}\text { Painful swelling for } 5 \text { years. } \\
\text { MRI: A soft-tissue mass, } 12 \times 8 \times 5 \mathrm{~cm}\end{array}$ & $\begin{array}{l}\text { Typical } \\
\text { S- } 100^{+}\end{array}$ & Surgical excision & $\begin{array}{l}\text { None } \\
(9 \text { months })\end{array}$ \\
\hline $\begin{array}{l}\text { Merritt } \\
\text { et al. (42), } 2019\end{array}$ & 56 & M & Left plantar & $\begin{array}{l}\text { Progressive, painful mass for } 5 \text { years. } \\
\text { MRI: A well-circumscribed, } \\
\text { subcutaneous mass, } 0.8 \times 0.6 \times 0.5 \mathrm{~cm}\end{array}$ & $\begin{array}{l}\text { Typical } \\
\text { S- } 100^{+}\end{array}$ & Surgical excision & $\begin{array}{l}\text { None } \\
(2 \text { months })\end{array}$ \\
\hline \multirow[t]{5}{*}{$\begin{array}{l}\text { Angelini } \\
\text { et al. (43), } 2019\end{array}$} & 57 & $\mathrm{~F}$ & $\begin{array}{l}\text { Right plantar } \\
\text { forefoot }\end{array}$ & $\begin{array}{l}\text { An oval-shaped nodule with pain } \\
\text { and paresthesia for } 1 \text { year, } 1 \times 1.5 \mathrm{~cm}\end{array}$ & Typical & Surgical excision & $\begin{array}{l}\text { None } \\
\text { (3 months) }\end{array}$ \\
\hline & 45 & M & $\begin{array}{l}\text { Right plantar } \\
\text { midfoot }\end{array}$ & $\begin{array}{l}\text { Right foot pain and swelling } \\
\text { 1st MRI: Synovial angiomatosis. } \\
\text { Symptoms returned after } 6 \text { months } \\
\text { surgical excision. }\end{array}$ & $\begin{array}{l}\text { 1st Surgical } \\
\text { excision: } \\
\text { Synovial } \\
\text { angiomatosis }\end{array}$ & Surgical excision & N/A \\
\hline & & & & $\begin{array}{l}\text { 2nd MRI: tumor-like tissue in the } \\
\text { plantar region of the foot suspected } \\
\text { for local recurrence }\end{array}$ & $\begin{array}{l}\text { 2nd Surgical } \\
\text { excision: } \\
\text { Typical } \\
\text { schwannoma }\end{array}$ & & \\
\hline & 58 & $\mathrm{~F}$ & Left foot & $\begin{array}{l}\text { Left foot pain for } 4 \text { years. } \\
\text { MRI: A mass arising from the sural } \\
\text { nerve near the lateral malleolus }\end{array}$ & Typical & Surgical excision & N/A \\
\hline & 35 & $\mathrm{~F}$ & $\begin{array}{l}\text { Right medial } \\
\text { ankle }\end{array}$ & $\begin{array}{l}\text { A slowly growing, poorly mobile, } \\
\text { painful mass for } 2 \text { years, Tinel+. } \\
\text { MRI: A fusiform lesion along the course } \\
\text { of the medial plantar nerve }\end{array}$ & Typical & $\begin{array}{c}\text { Surgical } \\
\text { enucleation }\end{array}$ & $\begin{array}{c}\text { None } \\
\text { (48 months) }\end{array}$ \\
\hline $\begin{array}{l}\text { Daniel } \\
\text { et al. }(44), 2019\end{array}$ & 61 & M & Left heel & $\begin{array}{l}\text { A sharp painful mass for } 7 \\
\text { years, progressively worse. Tinel+. } \\
\text { MRI: A hyperintense mass arising } \\
\text { from tibial nerve, } 2.2 \mathrm{~cm}\end{array}$ & $\begin{array}{l}\text { Typical } \\
\text { S-100+ }\end{array}$ & Surgical excision & $\begin{array}{l}\text { None } \\
(12 \text { months })\end{array}$ \\
\hline
\end{tabular}

F: Female; M: male; MRI: magnetic resonance imaging; N/A: not available. Typical schwannoma means, histologically, spindle cells arranged in palisading patterns in Antoni A zone and hypocellular regions in Antoni B zone.

patients' ages ranged from 8 to 84 years, with a mean and median of 47.4 and 51.5 years, respectively. The tumor size ranged from $0.38 \mathrm{~cm}$ to $14 \mathrm{~cm}$ in diameter, with an average of $3.3 \mathrm{~cm}$ and a median of $2.5 \mathrm{~cm}$. The tumor locations of 46 patients were retrieved, with $28(60.9 \%)$ being located on the left side. The tumors were mainly localized on the ankle $(14 / 46,30.3 \%)$, plantar aspect $(14 / 46,30.3 \%)$ and heel $(9 / 46$, $19.6 \%)$. Three tumors were found in the dorsal web of the interdigital spaces, one on the dorsum of the foot. Subanatomical locations on the foot were not specified in five patients. Out of the 49 patients, 29 were pre-surgically examined by magnetic resonance imaging (MRI). Typical MRI of schwannoma showed a well-circumscribed, round or oval mass with isointense or reduced signal intensity relative to surrounding skeletal muscle on T1-weighted images and significantly increased homogeneous or heterogeneous signal intensity on T2-weighted images (Figure 1). Fascicular sign (fascicular bundles in neurogenic tumors), target sign (centrally decreased with peripherally increased signal intensity) and split-fat sign (a rim of fat surrounding the tumor), reflecting different proportions of stromal tissues and tumor components (4), were described. Forty-eight out of 49 patients were selected to undergo surgical excision or enucleation, whereas one patient from our series opted for observation after biopsy due to the small size. No recurrences were reported, with a period of follow-up from 2 months to four years.

Grossly, schwannomas were usually round, ovoid, wellcircumscribed, solid masses. The cut surface was often gray to yellow, solid, glistening and well-encapsulated. Degenerative changes including cystic formation and hemorrhage were observed in some cases. Histopathologically, 

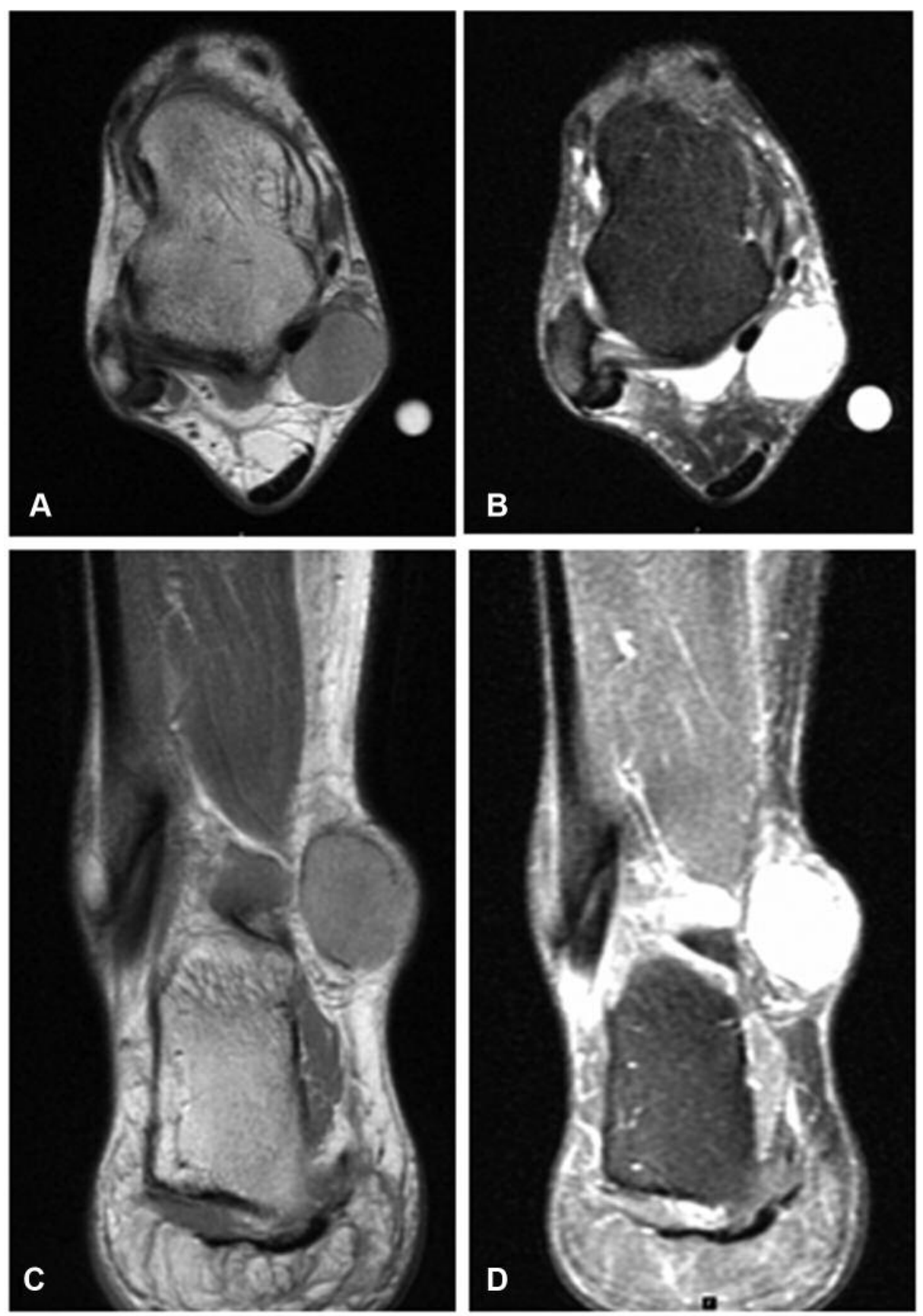

Figure 1. Magnetic resonance imaging of patient 1 showing a well-circumscribed, solid mass along the posteromedial aspect of the right ankle with iso-intensity signal relative to surrounding muscles on T1-weighted images (A, coronal view; $C$, sagittal view) and hyperintensity signal on T2weighted images (B: coronal view, D: sagittal view).

the majority of schwannomas exhibited typical biphasic morphology, composed of hypercellular Antoni A and hypocellular Antoni B zones. Antoni A zone consisted of elongated spindle cells with indistinct borders palisaded together, forming a Verocay body, while Antoni B presented reduced cellularity in a myxoid matrix with increased vascularization (Figure 2). Other variants, including plexiform and ancient types, were reported. One variant of schwannoma with 'ancient' changes in our series showed atypical nuclei with increased size and chromatin density. Immunostain showed strong positive cytoplasmic staining for S-100 but negative staining for alpha smooth actin ( $\alpha$ SMA) and CD34 


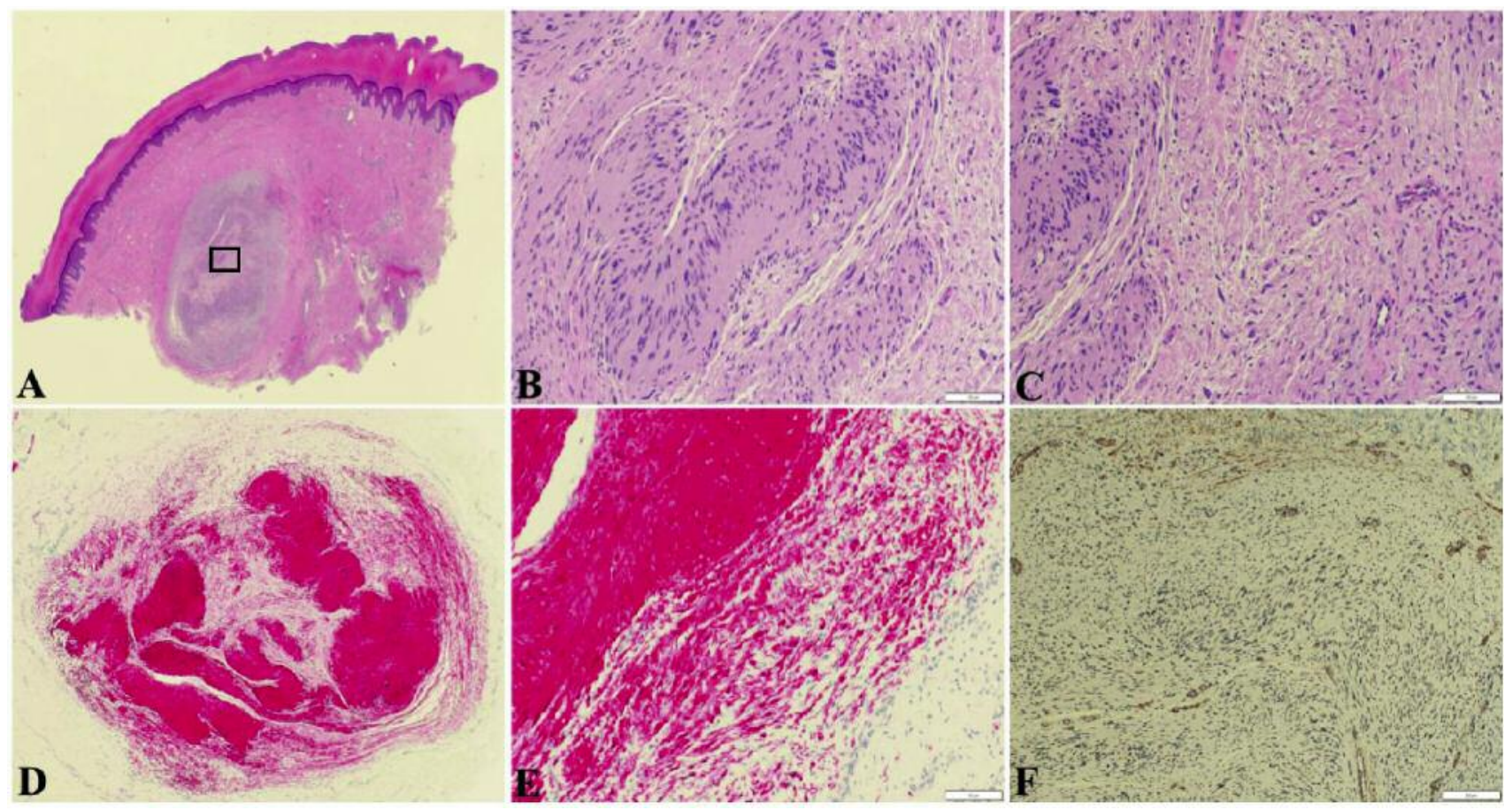

Figure 2. Microphotographs of hematoxylin and eosin and immunostained schwannoma on the heel of patient 2. A: A full view of the slide showing a well-circumscribed subcutaneous mass with hypercellular and hypocellular regions ( $\times 2)$. Inset shows areas described in B and C. B: The Antoni A zone, characterized by spindle Schwann cells arranged in palisading pattern (Verocay body) $(\times 200)$. C: The left third of the image shows the Verocay body in (A), while the right two-thirds shows Antoni B zone featuring hypocellularity in myxoid stroma $(\times 200)$. D: Microphotograph showing strong S-100 immunoexpression in tumor whereas surrounding stromal tissues were negative $(\times 5)$. E: Higher magnification of D showing S-100 cytoplasmic expression in spindle tumor cells where the encapsulated fibroblasts were negative $(\times 200)$. F: Microphotograph of immunostaining showing negative alpha-smooth muscle actin expression in spindle tumor cells but strong positive expression in vascular smooth muscle cells among tumor cells and surrounding stromal tissues, which served as an internal positive control $(\times 100)$.

in spindle cells (Figure 2). One of our patients showed coexistence of compound melanocytic nevus with underlying schwannoma, both neval cells of the nevus and spindle cells of the schwannoma exhibited positive staining for both S-100 and HMB-45.

\section{Discussion}

We presented seven cases of schwannoma of foot and ankle with its clinical manifestation, histopathology and treatment. Schwannoma accounted for $4.0 \%$ among 174 benign softtissue tumors involving the foot and ankle diagnosed in a 3 -year period at our Institute with 63 podiatrists. Ruggieri et al. reported that 14 schwannomas out of 189 benign softtissue tumors of the foot $(7.5 \%)$ were diagnosed at the Rizzoli Institute in an 18-year period between 1990 and 2007 (12). These data further support the observation that schwannoma on the foot and ankle is rare.

Clinically, schwannoma on the foot and ankle usually presents as a painful, movable, well-defined mass; weakness and paresthesia can be observed when associated nerves are affected. The symptoms are mainly related to the tumor location, especially on the sites of weight bearing or easily compressed regions including the plantar aspect and interdigital spaces. Tinel sign and tarsal tunnel syndrome can be elicited when the tibial nerve is evoked. Even though tumor can occur on any part of the foot, the most frequent locations were found to be the ankle, heel and plantar aspect of the foot (78.3\%). Occurrences in interdigital spaces and on dorsum of foot were uncommon. Both males and females were found to be equally affected. Interestingly, $60.9 \%$ of tumors occurred on the left foot. The modalities of pre-surgical diagnosis include X-ray radiography, ultrasound and MRI. Radiography usually does not reveal remarkable changes unless calcification noticed inside tumor tissue or bone tissues are involved. Ultrasound examination of schwannoma usually exhibits a round or ovoid, solid, well-delineated, hypoechoic homogenous mass and is useful for distinguishing between cystic and solid lesions. MRI is the most valuable tool for evaluating schwannoma, not only for location, size and texture, but also for relationships with 
Table III. Differential diagnosis of schwannoma from other tumor types.

\begin{tabular}{|c|c|c|c|}
\hline Tumor & Clinical presentation & Histology & Immunohistochemistry \\
\hline Ganglion cyst & $\begin{array}{l}\text { Fluid feeling on palpation, most on dorsal } \\
\text { surfaces and transillumination, aspiration } \\
\text { with yellow to clear viscous fluid }\end{array}$ & $\begin{array}{l}\text { Cyst surrounded by dense fibrous tissues } \\
\text { admixed with inflammatory cells, } \\
\text { no synovial or epithelial lining }\end{array}$ & \\
\hline Spindle cell lipoma & $\begin{array}{l}\text { Soft solid mass in the skin } \\
\text { and subcutaneous tissue }\end{array}$ & $\begin{array}{l}\text { Well-encapsulated spindle adipose } \\
\text { cells without atypia }\end{array}$ & $\mathrm{CD}_{3} 4^{+}, \mathrm{S}-100^{+}$ \\
\hline Neurofibroma & $\begin{array}{l}\text { Non-encapsulated, usually painless, } \\
\text { often multiple lesions throughout the body, } \\
\text { frequently seen in second and third decades }\end{array}$ & $\begin{array}{l}\text { Mixed cell types including Schwann cells, } \\
\text { perineurial-like cells, fibroblastic cells } \\
\text { and entrapped axons. Uniphasic, rare } \\
\text { palisading pattern. Variants including focal } \\
\text { and diffuse cutaneous, plexiform, intraneural } \\
\text { and pigmented neurofibroma }\end{array}$ & $\begin{array}{l}\mathrm{S}-100^{+}, \mathrm{SOX} 10^{+} \\
\mathrm{CD} 34^{+} \text {(focal), } \\
\text { factor } \mathrm{XIII}^{+} \text {(focal) }\end{array}$ \\
\hline Solitary fibrous tumor & $\begin{array}{l}\text { Usually occurring in older adults, } \\
\text { slow-growing and painless mass with } \\
\text { low rate of infiltration and metastasis }\end{array}$ & $\begin{array}{l}\text { Relatively bland and uniform spindle } \\
\text { cells within long, thin and parallel bands } \\
\text { of collagen in a 'patternless' arrangement }\end{array}$ & $\begin{array}{l}\mathrm{CD} 34^{+}, \mathrm{CD}_{99^{+}}, \\
\text {vimentin }^{+}, \text {desmin } \\
\text { S-, } \\
\text { S-100 } \\
\text { fusion gene, strong } \\
\text { nuclear STAT6 } \\
\text { expression }(45)\end{array}$ \\
\hline Fibroma & $\begin{array}{l}\text { Usually occurring along the plantar } \\
\text { medial arch within the central and/or } \\
\text { medial band of the plantar fascia }\end{array}$ & $\begin{array}{l}\text { Well encapsulated, spindled, oval or } \\
\text { round cells in fascicles }\end{array}$ & $\begin{array}{l}\text { Vimentin+, reticulin+ } \\
\text { collagen I+, } \\
\text { CD34-, } \alpha \text {-SMA- }\end{array}$ \\
\hline Leiomyoma & $\begin{array}{l}\text { Usually located in the skin and } \\
\text { subcutaneous tissue, frequently } \\
\text { seen in early adulthood }\end{array}$ & $\begin{array}{l}\text { Intersecting fascicles of benign smooth } \\
\text { muscle bundles without mitosis }\end{array}$ & $\begin{array}{l}\alpha-\mathrm{SMA}^{+}, \text {myosin }^{+} \\
\operatorname{desmin}^{+}, \mathrm{CD}^{-} 4^{-}\end{array}$ \\
\hline Neuroma & $\begin{array}{l}\text { Usually occurring in interdigital spaces, } \\
\text { painful with activity but relieved with rest } \\
\text { and massage, radiating to the adjacent toes }\end{array}$ & $\begin{array}{l}\text { Wavy nerve bundles surrounded } \\
\text { by dense fibrosis }\end{array}$ & $\mathrm{S} 100^{+}$ \\
\hline
\end{tabular}

NAB2-STAT6: NGFI-A binding protein 2 gene and signal transducer and activator of transcription 6; $\alpha$-SMA: alpha smooth muscle actin; CD: cluster of differentiation; SOX10: SRY-related HMG-box 10.

nerves and other surrounding anatomical structures. Typical MRI of schwannomas show isointense or reduced signal intensity relative to surrounding skeletal muscle on T1weighted images and significantly increased signal intensity on T2-weighted images.

Surgical excision or enucleation is the primary treatment option, even though for a smaller tumor observation can be opted for. Extreme care needs to be taken to dissect the tumor from associated nerves in order to preserve or restore nerve functions to a maximal level. Recurrence is extremely rare (less than 1\%) unless tumor tissue is incompletely excised $(4,17,18)$.

Morphologically, schwannoma of the foot and ankle is similar to that at other sites of the body. It is composed of an Antoni A zone with hypercellularity with palisaded spindle cells arranged in short bundles or fascicles, and an Antoni B zone with hypocellularity with vascu-larization in myxoid matrix. Immunostaining for S-100 is positive, whereas that for $\alpha$ SMA and CD34 is negative, which is useful for diagnosis and differential diagnosis. One of our patients had coexistence of compound melanocytic nevus and underlying schwannoma. It is not clear if there is any etiological relationship between these two lesions.
Schwannoma on the foot and ankle needs to be differentiated from other lesions, including ganglion cyst, neuroma, spindle-cell lipoma, neurofibroma, solitary fibrous tumor, fibroma, leiomyoma. Careful clinical and histopathological evaluation with immunohistochemistry can lead to an accurate diagnosis (Table III).

Trauma, Carney's complex, and neurofibromatosis type 1 or 2 may be associated with the development of schwannoma (19). One of our patients related frequently hitting the inside of his ankle against his chair at work as the probable cause of his tumor. In total, four [one of our cases plus three others (20-22)] out of 49 patients $(8.0 \%)$ reported minor trauma in the related areas before development of schwannoma. In one patient, schwannoma was associated with neurofibromatosis 1 (14), whereas no clinical presentation of neurofibromatosis for any of the other patients, suggesting that the majority of schwannomas involving the foot and ankle occur spontaneously.

In conclusion, schwannoma on the foot is a rare tumor. Both males and females are equally affected. It is histologically composed of palisaded spindle cells forming Antoni $\mathrm{A}$ and $\mathrm{B}$ zones. Ultrasound and MRI are good diagnostic tools for revealing tumor location, size, texture, 
involved nerves and relationships with surrounding muscular structures. Surgical excision is the primary treatment option with recurrence being rare.

\section{Financial Disclosure}

None reported.

\section{Conflicts of Interest}

None reported.

\section{Authors' Contributions}

Xingpei Hao: Study conceptualization, data collection, analysis and interpretation, writing and revising the article. David Levine: Patient care, data collection, analysis and interpretation, article revision. Joon Yim: Diagnosis, data collection, analysis and interpretation, article revision. Chenfeng Qi: Diagnosis, data collection, analysis and interpretation, article revision. Lee Firestone: Patient care, data collection, analysis and interpretation, article revision. Ian Beiser: Patient care, data collection, analysis and interpretation, article revision. Enzo Leone: Patient care, data collection, analysis and interpretation, article revision. Kirk Woelffer: Patient care, data collection, analysis and interpretation, article revision. Gene Mirkin: Study conceptualization, patient care, data collection, analysis and interpretation, coordination, article revision.

\section{References}

1 Verocay J: Zur kenntnis der "'neurofibrome". Beitr Pathol Anat 48: 60, 1910.

2 Masson P: Recklinghausen's neurofibromatosis, sensory neuromas and motor neuromas. In: International Press: New York, pp. 793, 1932.

3 Kransdorf MJ: Benign soft-tissue tumors in a large referral population: Distribution of specific diagnoses by age, sex, and location. Am J Roentgenol 164(2): 395-402, 1995. PMID: 7839977. DOI: 10.2214/ajr.164.2.7839977

4 Pilavaki M, Chourmouzi D, Kiziridou A, Skordalaki A, Zarampoukas $\mathrm{T}$ and Drevelengas A: Imaging of peripheral nerve sheath tumors with pathologic correlation: Pictorial review. Eur J Radiol 52(3): 229-239, 2004. PMID: 15544900. DOI: 10.1016/ j.ejrad.2003.12.001

5 Das Gupta TK, Brasfield RD, Strong EW and Hajdu SI: Benign solitary schwannomas (neurilemomas). Cancer 24(2): 355-366, 1969. PMID: 5796779. DOI: 10.1002/1097-0142 (196908) 24:2<355::aid-cncr2820240218>3.0.co;2-2

6 Knight DM, Birch R and Pringle J: Benign solitary schwannomas: A review of 234 cases. J Bone Joint Surg Br 89(3): 382-387, 2007. PMID: 17356155. DOI: 10.1302/0301-620X.89B3.18123

7 Kehoe NJ, Reid RP and Semple JC: Solitary benign peripheralnerve tumours. Review of 32 years' experience. J Bone Joint Surg Br 77(3): 497-500, 1995. PMID: 7744945.

8 Rosahl S, Bohr C, Lell M, Hamm K and Iro H: Diagnosis and management of vestibular schwannomas - an interdisciplinary challenge. Laryngorhinootologie 96(S01): S152-s182, 2017. PMID: 29036751. DOI: 10.1055/s-0042-122386
9 Halliday J, Rutherford SA, McCabe MG and Evans DG: An update on the diagnosis and treatment of vestibular schwannoma. Expert Rev Neurother 18(1): 29-39, 2018. PMID: 29088993. DOI: $10.1080 / 14737175.2018 .1399795$

10 Chou LB, Ho YY and Malawer MM: Tumors of the foot and ankle: Experience with 153 cases. Foot Ankle Int 30(9): 836841, 2009. PMID: 19755066. DOI: 10.3113/FAI.2009.0836

11 Azevedo CP, Casanova JM, Guerra MG, Santos AL, Portela MI and Tavares PF: Tumors of the foot and ankle: A singleinstitution experience. J Foot Ankle Surg 52(2): 147-152, 2013. PMID: 23333280. DOI: $10.1053 /$ j.jfas.2012.12.004

12 Ruggieri P, Angelini A, Jorge FD, Maraldi M and Giannini S: Review of foot tumors seen in a university tumor institute. J Foot Ankle Surg 53(3): 282-285, 2014. PMID: 24751209. DOI: 10.1053/j.jfas.2014.01.015

13 Toepfer A, Harrasser N, Recker M, Lenze U, Pohlig F, Gerdesmeyer L and von Eisenhart-Rothe R: Distribution patterns of foot and ankle tumors: A university tumor institute experience. BMC Cancer 18(1): 735, 2018. PMID: 30001718. DOI: $10.1186 / \mathrm{s} 12885-018-4648-3$

14 Carvajal JA, Cuartas E, Qadir R, Levi AD and Temple HT: Peripheral nerve sheath tumors of the foot and ankle. Foot Ankle Int 32(2): 163-167, 2011. PMID: 21288416. DOI: 10.3113/ FAI.2011.0163

15 Das Gupta TK and Chaudhuri PK: Tumors of the Soft Tissue. Second Edition. Appleton and Lange: Stanford, pp. 342-389, 1998.

16 Odom RD, Overbeek TD, Murdoch DP and Hosch JC: Neurilemoma of the medial plantar nerve: A case report and literature review. J Foot Ankle Surg 40(2): 105-109, 2001. PMID: 11324666.

17 Mangrulkar VH, Brunetti VA, Gould ES and Howell N: Unusually large pedal schwannoma. J Foot Ankle Surg 46(5): 398-402, 2007. PMID: 17761326. DOI: 10.1053/j.jfas.2007. 06.002

18 Torossian JM, Augey F, Salle M and Beziat JL: Giant foot schwannoma. Br J Plast Surg 54(1): 74-76, 2001. PMID: 11121325. DOI: $10.1054 /$ bjps.1999.3478

19 Hilton DA and Hanemann CO: Schwannomas and their pathogenesis. Brain Pathol 24(3): 205-220, 2014. PMID: 24450866. DOI: $10.1111 /$ bpa.12125

20 Buenger KM, Porter NC, Dozier SE and Wagner RF, Jr.: Localized multiple neurilemmomas of the lower extremity. Cutis 51(1): 36-38, 1993. PMID: 8419108.

21 Jacobson JM, Felder JM, 3rd, Pedroso F and Steinberg JS: Plexiform schwannoma of the foot: A review of the literature and case report. J Foot Ankle Surg 50(1): 68-73, 2011. PMID: 20833568. DOI: $10.1053 /$ j.jfas.2010.07.002

22 Judd T, Jones T and Thornberry L: Schwannoma of the posterior tibial nerve: Case study. J Am Podiatr Med Assoc 104(5): 539543, 2014. PMID: 25275747. DOI: 10.7547/0003-0538104.5.539

23 Zuckerman JD, Powers B, Miller JW and Lippert F: Benign solitary schwannoma of the foot. A case report and review of the literature. Clin Orthop Relat Res 228: 278-280, 1988. PMID: 3342580 .

24 Ikushima K, Ueda T, Kudawara I, Nakanishi K and Yoshikawa H: Plexiform schwannoma of the foot. Eur Radiol 9(8): 16531655, 1999. PMID: 10525885. DOI: 10.1007/s003300050904

25 Ritter SE and Elston DM: Cutaneous schwannoma of the foot. Cutis 67(2): 127-129, 2001. PMID: 11236222. 
26 Still GP: Neurilemoma of the medial plantar nerve: A case report. J Foot Ankle Surg 40(4): 236-239, 2001. PMID: 119246 85.

27 Marui T, Yamamoto T, Akisue T, Hitora T, Kawamoto T, Nagira $\mathrm{K}$, Yoshiya S and Kurosaka M: Neurilemmoma in the foot as a cause of heel pain: A report of two cases. Foot Ankle Int 25(2): 107-111, 2004. PMID: 14992711. DOI: 10.1177/1071100 70402500212

28 Ioannou M, Papanastassiou I, Iakowidou I, Kottakis S and Demertzis N: Plexiform schwannoma of the posterior tibial nerve: A case report. Cases J 2: 8392, 2009. PMID: 19918424. DOI: $10.4076 / 1757-1626-2-8392$

29 Kwok KB, Lui TH and Lo WN: Neurilemmoma of the first branch of the lateral plantar nerve causing tarsal tunnel syndrome. Foot Ankle Spec 2(6): 287-290, 2009. PMID: 20400427. DOI: $10.1177 / 1938640009349868$

30 Mendeszoon MJ, Cunningham N, Crockett RS and Kushner D: Schwannoma: A case report. Foot Ankle Online J 2(10): 4, 2009. DOI: $10.3827 /$ faoj.2009.0210.0004

$31 \mathrm{Li} \mathrm{XN}$, Cui JL, Christopasak SP, Kumar A and Peng ZG: Multiple plexiform schwannomas in the plantar aspect of the foot: Case report and literature review. BMC Musculoskelet Disord 15: 342, 2014. PMID: 25306531. DOI: 10.1186/14712474-15-342

32 Milnes HL and Pavier JC: Schwannoma of the tibial nerve sheath as a cause of tarsal tunnel syndrome--a case study. Foot 22(3): 243-246, 2012. PMID: 22560193. DOI: 10.1016/j.foot. 2012.03.005

33 Schweitzer KM Jr., Adams SB, Jr. and Nunley JA, 2nd: Multiple schwannomas of the posterior tibial nerve: A case series. Foot Ankle Int 34(4): 607-611, 2013. PMID: 23559618. DOI: $10.1177 / 1071100712470916$

34 Kellner CP, Sussman E, Bar-David T and Winfree CJ: Schwannomas of the foot and ankle: A technical report. J Foot Ankle Surg 53(4): 505-510, 2014. PMID: 23932119. DOI: $10.1053 /$ j.jfas.2013.05.007

35 Kallini JR and Khachemoune A: Schwannoma of the left foot: A brief overview with focus on associated clinical syndromes. J Am Podiatr Med Assoc 104(5): 535-538, 2014. PMID: 25275746. DOI: 10.7547/0003-0538-104.5.535

36 Hallahan K, Vinokur J, Demski S, Faulkner-Jones B and Giurini $\mathrm{J}$ : Tarsal tunnel syndrome secondary to schwannoma of the posterior tibial nerve. J Foot Ankle Surg 53(1): 79-82, 2014. PMID: 23954095. DOI: 10.1053/j.jfas.2012.12.020
37 Mohammed SA, Pressman MM, Schmidt B and Babu N: Case presentations and review of plexiform schwannoma in the foot. J Foot Ankle Surg 53(2): 179-185, 2014. PMID: 24556484. DOI: 10.1053/j.jfas.2013.12.020

38 Nishio J, Mori S, Nabeshima K and Naito M: Successful enucleation of large multinodular/plexiform schwannoma of the foot and ankle. Springerplus 4: 260, 2015. PMID: 26090307. DOI: $10.1186 / \mathrm{s} 40064-015-1087-3$

39 Min HJ, Kim KC, Jun SH and Lee YG: Schwannomatosis on a single foot: A case report. Foot Ankle Spec 8(3): 226-229, 2015. PMID: 25547315. DOI: 10.1177/1938640014565047

40 Albert P, Patel J, Badawy K, Weissinger W, Brenner M, Bourhill I and Parnell J: Peripheral nerve schwannoma: A review of varying clinical presentations and imaging findings. J Foot Ankle Surg 56(3): 632-637, 2017. PMID: 28237565. DOI: 10.1053/j.jfas.2016.12.003

41 Muratori F, De Gori M, Campo FR, Bettini L, D'Arienzo A, Scoccianti G and Capanna R: Giant schwannoma of the foot: A case report and literature review. Clin Cases Miner Bone Metab 14(2): 265-268, 2017. PMID: 29263749. DOI: $10.11138 / \mathrm{ccmbm} /$ 2017.14.2.265

42 Merritt Gt, Ramil M, Oxios A and Rushing C: Schwannoma of the plantarmedial aspect of the foot: A case report. Foot 39: 8587, 2019. PMID: 30981128. DOI: 10.1016/j.foot.2019.03.005

43 Angelini A, Bevoni R, Biz C, Cerchiaro MC, Girolami M and Ruggieri P: Schwannoma of the foot: Report of four cases and literature review. Acta Biomed 90(1-s): 214-220, 2019. PMID: 30715028. DOI: 10.23750/abm.v90i1-S.8079

44 Daniel M, Waters D, Chen C and Brouyette N: Posterior tibial nerve schwannoma in a multiple myeloma patient: A case report. SAGE Open Med Case Rep 7: 2050313x19838441, 2019. PMID: 31007915. DOI: 10.1177/2050313X19838441

45 Yoshida A, Tsuta K, Ohno M, Yoshida M, Narita Y, Kawai A, Asamura $\mathrm{H}$ and Kushima R: Stat6 immunohistochemistry is helpful in the diagnosis of solitary fibrous tumors. Am J Surg Pathol 38(4): 552-559, 2014. PMID: 24625420. DOI: 10.1097/ PAS.0000000000000137
Received July 23, 2019

Revised August 8, 2019

Accepted August 9, 2019 